\title{
Application of the BLT equation for radiation field calculaton for double transmission line
}

\author{
Hongzhou XU \\ PLA 91550 \\ Unit 94 \\ Dalian, China \\ e-mail: clare911@sina.com
}

\begin{abstract}
By the excitation of the electromagnetic signal, double transmission line will inducing a voltage and current, thereby creating the space radiation field. Based on infinitesimal current source radiation field and by the convenience of the terminal response aspects of solving transmission line of BLT equation, the double transmission line is derived and gets the analytical solution.
\end{abstract}

Keywords-BLT equation; double transmission line; radiation field; analytical solution

\section{INTRODUCTION}

By the excitation of the electromagnetic signal, double transmission line will inducing a voltage and current, thereby creating the space radiation field. The paper based on infinitesimal current source radiation field and by the convenience of the terminal response aspects of solving transmission line of BLT equation, the double transmission line is derived and get the analytical solution of frequency domain.

\section{RADIATION FIELD OF DOUBLE TRANSMISSION LINE}

Figure 1 shows the L Length simplified model of positive propagation voltage wave $V^{+}(x)$ of double transmission line traveling wave radiation field, the corresponding negative propagation voltage wave $V^{-}(x)$ is also exist, but won't mark here. The positive propagation voltage wave $V^{+}(x)$ and negative propagation voltage wave $V^{-}(x)$ are decided by the excitation source. Let's show double guide line up going wave in its surroundings produced by the radiation field by the transmission line theory by using terminal load in incident voltage wave $V^{\text {he }}$.

Figure 1 shows us that if assume that radiation field and the $X$ axis negative Angle for $\psi$ which distance origin of coordinates the distance to $r_{0}$ and $r_{0}$ is sufficiently large to guarantee the radiation field for plane wave. For positive propagation voltage wave $V^{+}(x)$, The corresponding current wave is $I^{+}(x)=V^{+}(x) / Z_{c}, Z_{c}$ is the characteristics of the transmission line impedance, and two conducting online relatively independent current $I_{1}$ and $I_{2}$ defined as $I_{2}=-I_{1}$.

\author{
Wanjin WANG \\ PLA 91550 \\ Unit 94 \\ Dalian, China
}

e-mail: wwj.1982-2002@163.com

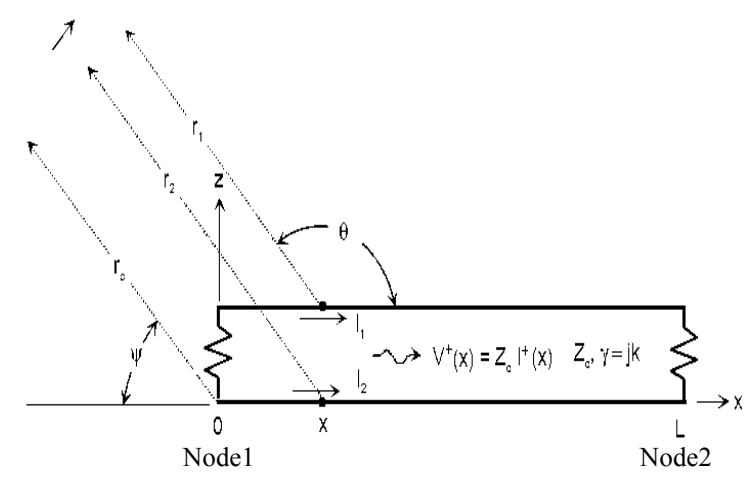

Figure 1. Model of transmission line traveling wave radiation

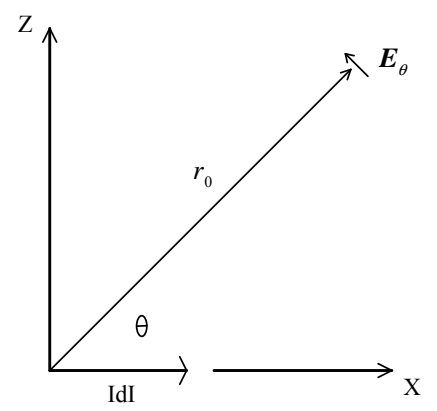

Figure 2. Radiation field of infinitesimal current source

Before solving the radiation field produced by current wave, let's review the radiation situation produce by infinitesimal current source. As shown in Figure 2, on the far side current source IdI generated for electric field is:

$$
\boldsymbol{E}_{\theta}=\frac{\mathrm{j} \omega \mu_{0}}{4 \pi r_{0}} \sin \theta \boldsymbol{I} \boldsymbol{d I} \mathrm{e}^{-\mathrm{j} \sigma_{\theta}}
$$

Because $\boldsymbol{E}_{\theta}$ and $\boldsymbol{E}_{f}$ in opposite directions and $\sin \theta=\sin \psi$, so we can get that the two infinitesimal current produced by the radiation field of the double transmission line at $\mathrm{x}$ place:

$$
d \boldsymbol{E}_{i}=-\frac{\mathrm{j} \omega \mu_{0}}{4 \pi} \sin \psi\left(I_{1}(x) \frac{\mathrm{e}^{-\mathrm{j} \omega_{\mathrm{i}}}}{r_{1}}+I_{2}(x) \frac{\mathrm{e}^{-\mathrm{j} \kappa_{2}}}{r_{2}}\right)
$$

The current wave on the transmission line can be expressed by incident voltage wave on node 2 : 


$$
I_{1}(x)=-I_{2}(x)=\frac{V_{2}^{\ln c}}{Z} \mathrm{e}^{-\gamma(x-1)}
$$

And in the far sites:

$$
\begin{aligned}
\frac{1}{r_{1}} & \approx \frac{1}{r_{2}} \approx \frac{1}{r_{0}} \\
\mathrm{e}^{-\mathrm{j} k r_{1}} & =\mathrm{e}^{-\mathrm{j} k\left(r_{0}+\cos \psi-d \sin \psi\right)} \\
\mathrm{e}^{-\mathrm{j} k r_{2}} & =\mathrm{e}^{-\mathrm{j} k\left(r_{0}+x \cos \psi\right)}
\end{aligned}
$$

Put Eq.3 Eq.6 into Eq.2, we can get:

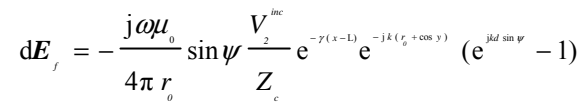

Because $e^{j k \sin \psi}-1 \approx j k d \sin \psi$, Eq.7 can be transform to:

$$
\mathrm{d} \boldsymbol{E}_{i}=\frac{k^{2} d}{4 \pi} \frac{Z_{o}}{Z_{c}} \frac{\mathrm{e}^{-j \omega_{o}}}{r_{0}} \sin ^{2} \psi V_{2}^{\operatorname{lnc} c} \mathrm{e}^{j k L} \mathrm{e}^{-j k(1+\cos y) x}
$$

There into $k=\frac{\omega}{c}, Z_{0}=c \mu_{0}$.

According to integral of Eq1 on $0 \sim \mathrm{L}$ we can get:

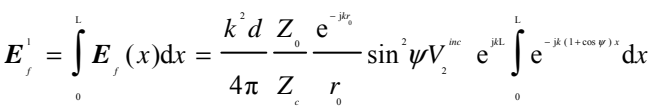

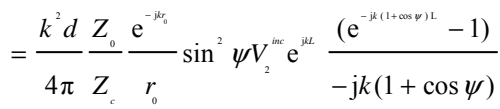

$$
\begin{aligned}
& =\frac{j k d}{4 \pi} \frac{Z_{0}}{Z} \frac{\mathrm{e}^{-j k_{i}}}{r_{0}} V_{2}^{i m c} \mathrm{e}^{j k L} \frac{\sin ^{2} \psi}{(1+\cos \psi)}\left(\mathrm{e}^{-j k(1+\cos \psi) L}-1\right)
\end{aligned}
$$

Due to the terminal load in travelling wave will also produce radiation field, Eq.9 are not the total radiation field produced by positive travelling wave, the radiation condition of traveling wave of terminal load place is also need to be considered. As shown in Figure 3:

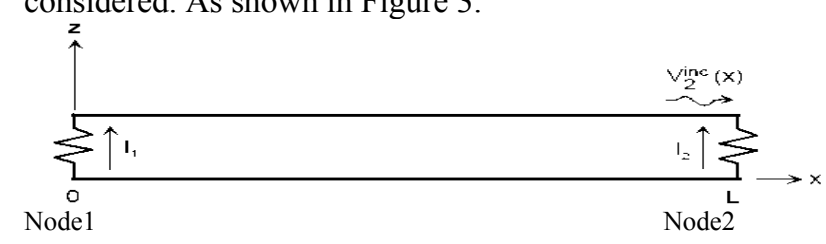

Figure 3. Traveling wave of terminal load place

$I_{1}$ At $x=0$ and $I_{2}$ at $x=\mathrm{L}$ can be expressed by incident voltage $V_{2}^{\text {inc }}$ at node2:

$$
\begin{aligned}
& I_{1}=\frac{V_{2}^{\text {inc }}}{Z_{c}} \mathrm{e}^{\text {jat. }} \\
& I_{2}=-\frac{V_{2}^{\text {inc }}}{Z}
\end{aligned}
$$

Use Eq.1, we can get the current radiation field of terminal load place:

$$
\begin{aligned}
& \boldsymbol{E}_{,}^{2}=-\frac{\mathrm{j} \omega \mu_{0}}{4 \pi r} \cos \psi \mathrm{e}^{-\mathrm{j} \mathrm{j}_{\mathrm{r}}}\left(I_{1} d+I_{2} d \mathrm{e}^{-\mathrm{j} \text { Heoser }}\right) \\
& =\frac{j k d}{4 \pi} \frac{Z_{0}}{Z} \frac{\mathrm{e}^{-j k_{0}}}{r_{0}} V_{2}^{\text {inc }} \mathrm{e}^{j \mu t} \cos \psi\left(\mathrm{e}^{-j k(1+\cos \psi) \mathrm{L}}-1\right)
\end{aligned}
$$

By Eq. 9 and Eq. 12 we can get that the total radiation field of positive propagation current wave is:

$$
\boldsymbol{E}^{+}=\boldsymbol{E}_{f}^{\prime}+\boldsymbol{E}_{f}^{2}=\frac{j k d}{4 \pi} \frac{Z_{0}}{Z_{c}} \frac{\mathrm{e}^{-j \sigma_{c}}}{r_{0}} V_{2}^{i m c} \mathrm{e}^{j k L}\left(\mathrm{e}^{-j k(1+\cos \varphi) L}-1\right)
$$

Define negative spread coupling function:

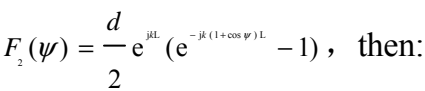

$$
\boldsymbol{E}^{+}=\frac{j k}{2 \pi} \frac{Z_{0}}{Z} \frac{\mathrm{e}^{-j \psi_{0}}}{r} F_{2}(\psi) V_{2}^{\text {mec }}
$$

By the same token, define forward propagation coupling function: $F_{1}(\psi)=\frac{d}{2}\left(\mathrm{e}^{i(1-\cos \psi) !}-1\right)$ then get that the total radiation field of negative propagation current wave is:

$$
\boldsymbol{E}^{-}=\frac{\mathrm{jk}}{2 \pi} \frac{Z_{0}}{Z_{c}} \frac{\mathrm{e}^{-j r_{0}}}{r_{0}} F_{1}(\psi) V_{1}^{\text {inc }}
$$

By Eq.14 and Eq.15 we can get that the total radiation field of positive propagation current wave and negative spread current wave propagation current wave is:

$$
\mathrm{E}=\mathrm{E}^{+}+\mathrm{E}^{-}=\frac{\mathrm{j} k}{2 \pi} \frac{Z_{0}}{Z_{c}} \frac{\mathrm{e}^{-j k_{c}}}{r_{0}}\left(F_{1}(\psi) V_{1}^{i m c}+F_{2}(\psi) V_{2}^{i m c}\right)
$$

\section{RADIATION FIELD ANALYTICAL SOLUTION}

Through the transmission line theory and infinitesimal current source radiation field, we can get the general expression of double transmission line radiation field. By Eq.16 we can find that double transmission line is decided by the incident wave voltage $V_{1}^{\text {inc }}$ and $V_{2}^{\text {inc }}$ of radiation field by its terminal place. Therefore, it is necessary to get incident voltage wave before solving double transmission line of the radiation field analytical solution. Next, we will use the reasoning way of BLT equation for radiation field calculation for double transmission line to get the incident wave voltage $V_{1}^{\text {inc }}$ and $V_{2}^{\text {inc }}$, and finally get the analytical solution of.

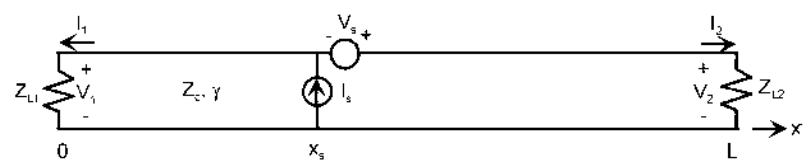


Node1 Excitation point Node2

Figure 4. Model of double conductor transmission line

In reference [3], Figure 4 shows that double conductor line terminal in incident voltage wave is expressed as the matrix form:

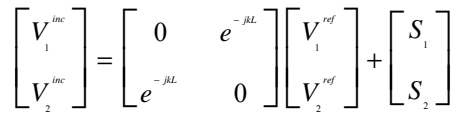

Among them, $V_{1}^{\text {ref }}$ and $V_{2}^{\text {ref }}$ is the incident voltmeter of node 1 and node 2 place in Figure $4, V_{1}^{\text {ref }}$ and $V_{2}^{\text {ref }}$ is the reflected voltage wave of node 1 and node 2 place in Figure 4, vector $[\mathrm{S}]$ is expressed by lumped source voltage and current source.

$$
[S]=\left[\begin{array}{c}
S_{1} \\
S_{2}
\end{array}\right]=\left[\begin{array}{c}
-\frac{1}{2}\left(V_{s}-Z_{c} I_{s}\right) e^{-j x_{s}} \\
\frac{1}{2}\left(V_{s}+Z_{c} I_{s}\right) e^{-\mu\left(L\left(L-x_{s}\right)\right.}
\end{array}\right]
$$

At each end of the transmission line, the relationship between reflected voltage wave and the incident voltage wave is:

$$
V^{\text {ref }}=\rho V^{\text {inc }}
$$

$\rho$ is the frequency domain voltage reflection coefficient, and transmission line terminal impedance $Z_{L}$ and characteristic impedance $Z$ related, defined as:

$$
\rho=\frac{Z_{L}-Z_{c}}{Z_{L}+Z_{c}}
$$

By Eq.18 we know that, the reflection in the voltage wave vector can by incident voltage wave vector Eq.17 can be expressed as:

$$
\left[\begin{array}{c}
V_{1}^{\text {eff }} \\
V_{2}^{\text {eff }}
\end{array}\right]=\left[\begin{array}{cc}
\rho_{1} & 0 \\
0 & \rho_{2}
\end{array}\right]\left[\begin{array}{c}
V_{1}^{\text {nec }} \\
V_{2}^{\text {nec }}
\end{array}\right]
$$

Among them, $\rho_{1}$ and $\rho_{2}$ are the terminal voltage reflection coefficient of double conductor line.

Put Eq.19 into Eq.17, and solution about the incident voltage wave vector equation, we can get:

$$
\left[\begin{array}{l}
V_{1}^{\text {mec }} \\
V_{2}^{\text {mec }}
\end{array}\right]=\left[\left[\begin{array}{ll}
1 & 0 \\
0 & 1
\end{array}\right]-\left[\begin{array}{cc}
0 & e^{-\mu \omega} \\
e^{-\mu \omega} & 0
\end{array}\right]\left[\begin{array}{cc}
\rho_{1} & 0 \\
0 & \rho_{2}
\end{array}\right]\right]^{-1}\left[\begin{array}{l}
S_{1} \\
S_{2}
\end{array}\right]
$$

By Eq. 19 we can get the load voltage vector is:

$$
\left[\begin{array}{l}
V_{1} \\
V_{2}
\end{array}\right]=\left[\begin{array}{c}
V_{1}^{\text {inc }} \\
V_{2}^{\text {inc }}
\end{array}\right]+\left[\begin{array}{c}
V_{1}^{\text {nff }} \\
V_{2}^{\text {nff }}
\end{array}\right]=\left[\begin{array}{cc}
1+\rho_{1} & 0 \\
0 & 1+\rho_{2}
\end{array}\right]\left[\begin{array}{l}
V_{1}^{\text {inc }} \\
V_{2}^{\text {inc }}
\end{array}\right]
$$

Put Eq.20 into Eq.21, get the BLT equation of frequency domain in double conductor transmission line voltage is:

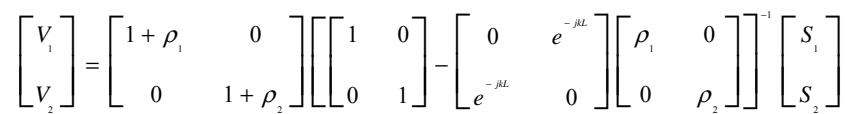

From the above results we can know, put Eq.20 into Eq.16 can get the frequency domain analytic solution of double guide line radiation field is:

$$
\boldsymbol{E}=\left(1-\rho_{1} \rho_{2} e^{-2 j k L}\right)^{-1}\left[F_{1}(\psi)\left(\rho_{2} e^{-j k L} S_{2}+S_{1}\right)+F_{2}(\psi)\left(\rho_{1} e^{-j k L} S_{1}+S_{2}\right)\right]
$$

\section{CONCLUDING REMARKS}

This paper is based on differential current source radiation field, did formula derivation with transmission line theory of double guide transmission line traveling wave radiation field. Released the expression of radiation field. We can see through the expression that double guide transmission line traveling wave radiation field is determined by the terminal incident voltage wave of only. So it absolutely necessary to get the double guide transmission line terminal branch of the incident voltage wave if you want to analyze the upward wave radiation field. Here perfectly combines transmission line theory and double guide transmission line BLT equation, getting the specific form of double guide transmission line terminal branch of the incident voltage wave by BLT equation thought. Meanwhile, combines it with double guide transmission line traveling wave radiation field general expression, to figure out double guide transmission line traveling wave radiation field analytical expression, which is also called frequency domain solution.

\section{REFERENCES}

[1] F. M. Tesche. EMC Analysis Methods and Computational Models[M]. New York: Wiley, 1997.

[2] C. E. Baum, T. K Liu, F. M. Tesche. On the analysis of general multiconductor transmission line networks [J]. Interaction Note 350, 1978, (11):263-293.

[3] F. M. Tesche, C. M. Butler. On the addition of EM field propagation and coupling effects in the BLT equation[J]. Interaction Note 588, 2003, (13):1-43.

[4] Guo Huiping, Liu Xueguan. Electromagnetic field and wave [M]. Press of Xian university of electronic science and technology,2003.

[5] K.S.H. Lee. EMP Interaction: Principles, Techniques and Reference Data[M]. New York: Taylor and Francis, 1989.

[6] T. K. Liu, F. M. Tesche. Analysis of antennas and scatters with nonlinear loads[J]. IEEE Trans. Antennas Propag,1976, 24(2):131139

[7] F. M. Tesche. Extension of the BLT Equation for Transient Solutions for Transmission Line Systems with Nonlinear 